\title{
Stochastic programming framework for Lithuanian pension payout modelling
}

\author{
Audrius Kabašinskas ${ }^{1, *}$, Kristina Šutienè $\dot{1}^{1}$, Eimutis Valakevičius ${ }^{1}$ and \\ Francesca Maggioni ${ }^{2}$ \\ ${ }^{1}$ Department of Mathematical Modeling, Kaunas University of Technology \\ Studentu 50-141, 51368 Kaunas, Lithuania \\ E-mail: 〈\{audrius.kabasinskas, kristina.sutiene, eimutis.valakevicius\}@ktu.lt〉 \\ ${ }^{2}$ Department of Management, Economics and Quantitative Methods, \\ University of Bergamo \\ Via dei Caniana 2, 24127 Bergamo, Italy \\ E-mail: 〈francesca.maggioni@unibg.it〉
}

\begin{abstract}
The paper provides a scientific approach to the problem of selecting a pension fund by taking into account some specific characteristics of the Lithuanian Republic (LR) pension accumulation system. The decision making model, which can be used to plan a long-term pension accrual of the Lithuanian Republic (LR) citizens, in an optimal way is presented. This model focuses on factors that influence the sustainability of the pension system selection under macroeconomic, social and demographic uncertainty. The model is formalized as a single stage stochastic optimization problem where the long-term optimal strategy can be obtained based on the possible scenarios generated for a particular participant. Stochastic programming methods allow including the pension fund rebalancing moment and direction of investment, and taking into account possible changes of personal income, changes of society and the global financial market. The collection of methods used to generate scenario trees was found useful to solve strategic planning problems.
\end{abstract}

Key words: Pension modelling, scenario tree, long-term strategy, single stage stochastic programming

Received: October 4, 2014; accepted: December 29, 2014; available online: December 30, 2014

\section{Introduction}

In Lithuania, after the declaration of independence in 1990 and up to 2004, the pension system was governed by the Ministry of Social Security. A private pension fund was also available. However, only a small part of population could afford it.

${ }^{*}$ Corresponding author. 
In 2004, a reform was introduced allowing any citizen to transfer part of their personal income taxes (reported in Table 1) to a private pension fund (called pillar II of the system).

\begin{tabular}{|l|r|r|r|r|r|r|r|r|r|r|}
\hline Year & 2004 & 2005 & 2006 & 2007 & 2008 & 2009 & 2010 & 2011 & 2012 & 2013 \\
\hline Payment, \% & 2.5 & 3.5 & 4.5 & 5.5 & 5.5 & 2.0 & 2.0 & 2.0 & 1.5 & 2.5 \\
\hline
\end{tabular}

Table 1: Payments to pillar II pension funds in the period 2004-2013.

During this period, it was consequently possible for the participant either to choose complete dependence on the social security system or transfer part of the risk and taxes to one's private pension fund. The payout from the first one (called pillar I) is fixed for all citizens and depends on governmental policy, economic situation of the country, duration of employment during working life, salary, etc. Currently, that is approximately $250 € /$ month. The payout from the private pension fund is supported by the social security pension. This means that if the participant has selected the second option, one's pension is composed of the following two parts:

- Part of social security pension (pillar I),

- Payout from the pillar II pension fund.

Pillar III is optional, and anyone can have it. However, pillar III is expensive and the participant must have salary above the country's average to afford it. These expenses are related to the fact that payments to pillar III funds are made by the participants after they pay taxes ( $\sim 30 \%$ of salary).

In 2014 the pension system was reformed. Nevertheless, the payout structure remains the same, i.e., pillars I, II and III are supported, and now pillar II has more choices for payments to the fund.

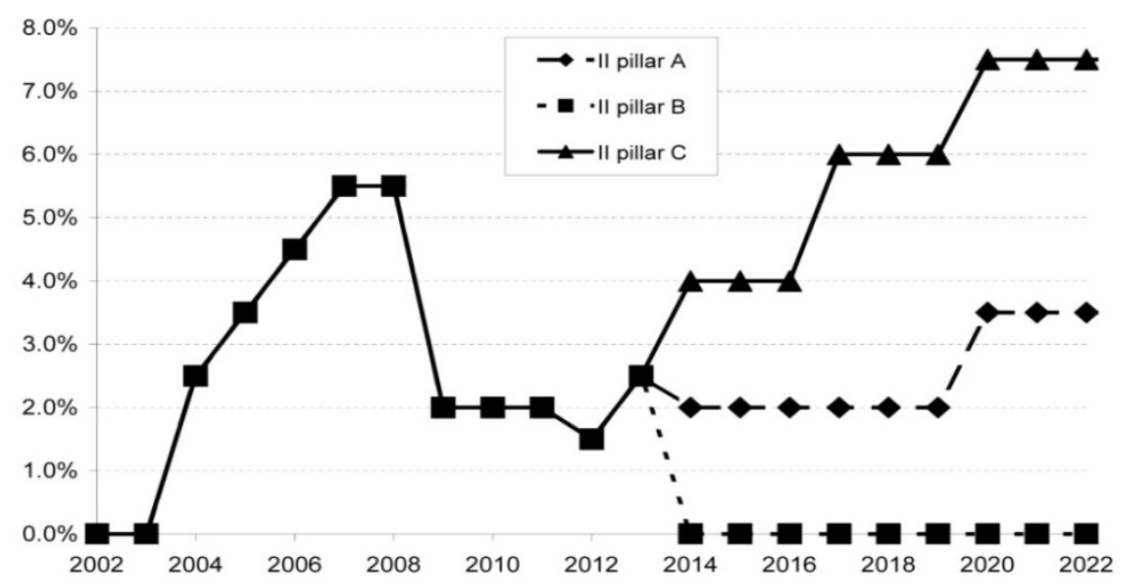

Figure 1: Planned payments to pillar II pension funds in the period 2004-2020. 
The possible choices are the following:

- the same as before (2\%). In 2020, this payment will be changed to $3.5 \%$ of salary before taxes (A, Figure 1).

- the participant may get back $2 \%$, add additional $1 \%$ of one's salary and receive the bonus of $1 \%$ from the average salary in Lithuania $(\sim 6$ $€ /$ month). In 2016 , the payment structure will be changed to $2+2+2 \%$. In 2020, it will be changed to $3.5+2+2 \%$ (C, Figure 1).

- the participant can also decide to stop accumulating in pillar II funds and remain only with pillar I (B, Figure 1).

Currently, there are 24 pension funds for pillar II of the system and some additional funds for pillar III. Funds are grouped into 4 classes:

- Conservative ( $0 \%$ stock);

- Small stock funds (less than $30 \%$ of stock);

- Medium stock funds (less than $70 \%$ of stock);

- Stock funds (up to $100 \%$ of stock).

The current structure of the funds market [1] is given in Table 2 .

\begin{tabular}{|l|c|r|r|r|r|}
\hline \multicolumn{1}{|c|}{ Fund type } & \multirow{2}{*}{$\begin{array}{c}\text { Number of } \\
\text { funds } \\
\text { available }\end{array}$} & \multicolumn{2}{|c|}{$\begin{array}{c}\text { Number of } \\
\text { participants }\end{array}$} & \multicolumn{2}{c|}{$\begin{array}{c}\text { Value of } \\
\text { managed assets }\end{array}$} \\
\cline { 3 - 6 } & & & $\%$ & \multicolumn{1}{l}{$\begin{array}{c}\text { LTL, } \\
\text { millions }\end{array}$} & $\%$ \\
\hline Conservative & 9 & 110,525 & 9.74 & 680.06 & 11.45 \\
\hline Small stock & 4 & 282,919 & 24.94 & $1,553.74$ & 26.15 \\
\hline Medium stock & 10 & 601,365 & 53.02 & $3,099.55$ & 52.17 \\
\hline Stock & 5 & 139,501 & 12.30 & 607.53 & 10.23 \\
\hline Total & 28 & $1,134,310$ & 100 & $5,940.88$ & 100 \\
\hline
\end{tabular}

Table 2: Funds market structure in Quarter 4 of 2013.

As one can see, the most popular group is medium stock with $53.02 \%$ of participants and the market capitalization of $52.17 \%$.

\section{Related work}

Nowadays, because of the reduction of the birth rate and the growth of life expectancy, the pension system has been reformed in many countries around the world. Lithuania is not an exception, since its social insurance is based on the solidarity principle, i.e., social security contributors are real-time finance social security beneficiaries $[18,3]$. The points of the previous pension system were examined by a number of Lithuanian scientists $[13,8]$. 
From a mathematical point of view, pillar II was the most difficult and hard to predict because it depends on the decisions made by the government. Since 2014, the Lithuanian system has become more complex, because two pension systems as pillar II are available now: one is old and the other is new.

This problem can be formulated as a decision making model, which can be used to plan the long-term Lithuanian citizen's pension accrual in an optimal way.

Because of the advantages of stochastic programming methodology, its application in the management of personal finances becomes a focus of current research. The aspects considered are the following: analysis of asset allocation into investments as a loss avoidance function for the objective formulation [4], comparison of investment opportunities that are offered to invest into retirement and life insurance alternatives [5], and the integration of stochastic and dynamic programming methods to study the personal finance and retirement pension [12]. Stochastic programming methodology also has the advantage to provide the opportunity for rebalancing the decisions in the longterm planning period and describing them in the algebraic form.

Stochastic programming deals with the problems where not all parameters are known with certainty, and the solutions which involve decisions made on the basis of a temporal flow of information. Such models involve a discrete representation of random events by means of a scenario tree; this technique facilitates a description of real problems including those of very large dimensions.

Approaches of scenario tree generation are based on various principles [2,9,10,11]: (a) bound-based constructions, (b) Monte-Carlo schemes or Quasi Monte-Carlo based methods, (c) EPVI-based sampling and reduction within decomposition schemes, (d) moment-matching principle, (e) probability metric based approximations. In general, the approaches have to be adapted to the problem considered in research.

\section{Methodology}

\subsection{Data analysis}

The return of the funds value obtained from historical data was fitted to some known probability distributions (normal, $\alpha$-stable). All analysed data are nonnormally distributed [19]; however, most of it is $\alpha$-stable distributed with $\alpha$ close to 1.5. Parameter $\beta$ in almost all cases is negative, and this indicates that the expected return probably will be less than the mean return. 
The retirement age in Lithuania is currently 61 for women and 63 for men. Since 2026, this age will be equal to 65 years for both genders. The work record $(w r)$ is the most important parameter for the pension accrual. Usually, it is necessary to have 30 years of work recorded to get a higher social security pension. However, if a citizen starts working immediately after the university or college graduation (or even earlier), one's work record will be $40-45$ years. The minimal employment period is 30 years to get some bonuses from social security funds.

The average salary before taxes (gross salary) in Quarter 4 of 2013 was $677.77 € /$ month. The complete list of the average and minimal gross salaries in the period 2004-2013 is given in Table 3 .

\begin{tabular}{|l|c|c|c|c|c|c|c|c|c|c|}
\hline Year & 2004 & 2005 & 2006 & 2007 & 2008 & 2009 & 2010 & 2011 & 2012 & 2013 \\
\hline $\begin{array}{l}\text { Average } \\
\text { gross } \\
\text { salary }\end{array}$ & 332.86 & 369.61 & 433.18 & 522.01 & 623.18 & 595.46 & 575.79 & 592.53 & 615.09 & 645.85 \\
\hline $\begin{array}{l}\text { Minimal } \\
\text { salary }\end{array}$ & 139.98 & 152.05 & 166.53 & 188.25 & 231.70 & 231.70 & 231.70 & 231.70 & 237.73 & 289.62 \\
\hline
\end{tabular}

Table 3: Average gross and minimal salaries in Lithuania in the period 2004-2013 $(€ /$ month) $[6,7]$.

Minimal salary is controlled by the government and currently (in 2014) it is equal to $289.62 € /$ month ( $\sim 20 \%$ of population has such salary). In our model, we will use various yearly salary increment rates current salary-initial salary (depending on the scenario), i.e., $0 \%, 1 \%, 2 \%, \ldots, 37 \%$. initial salary

\subsection{Stochastic programming framework (linear single stage formulation)}

We consider the following single-stage stochastic optimization linear problem $[2$, $14,15,16]$

$$
\begin{aligned}
\max - & \left(c_{2} y_{2}+c_{3} y_{3}\right)+\sum_{s=1}^{K} p_{s}\left(y_{1} \cdot x_{I, S}+y_{2} \cdot\left(x_{I I, S}+0.7 x_{I, S}\right)+y_{3} \cdot x_{I I I, S}\right) \\
& \left\{\begin{array}{l}
y_{1}+y_{2}=1 \\
y_{1} \cdot x_{I, s}+y_{2} \cdot\left(x_{I I, s}+0.7 x_{I, S}\right)+y_{3} \cdot x_{I I I, S}>0.7 \cdot M S_{s} \\
y_{1}, y_{2}, y_{3} \in\{0 ; 1\}
\end{array}\right.
\end{aligned}
$$

with decision variables $y_{1}, y_{2}, y_{3} \in\{0 ; 1\}$, which are set to 0 if the respective pillar is not used, and to 1 if it is used. 
Stochastic parameters are given by $x_{I, S}, x_{I I, S}$ and $x_{I I I, S}$ from pillars I, II and III, where $s$ is a scenario index, the mean salary of a participant for some period before retirement, $M S_{S}$, and $p_{S}$ is the scenario probability.

Payouts $x_{I, S}, x_{I I, S}$ and $x_{I I I, S}$ depend on the following parameters: $x_{I, S}=x_{I, s}\left(w r, M S_{s}\right), \quad x_{I I, s}=x_{I I, s}\left(w r, s i_{s}, f r_{s}, S_{s}, a, \gamma\right), \quad x_{I I I, s}=x_{I I I, s}\left(f r_{s}, P_{s}, a\right)$ and they are expected to satisfy the following relation $x_{I I, s}>0.3 \cdot x_{I, s}$, where $w r$ is work record, $s i_{s}$ is salary increment (expected to be non-negative), $S_{s}$ is the salary of a participant; $-\delta<f r_{s}<+\infty$ annual return on the pension fund (from recent research [19] it is known that $\operatorname{fr}_{s} \sim S_{\alpha}(\sigma, \beta, \mu)$ or $f r_{s} \sim \operatorname{mix} S(\alpha, \sigma, \beta, \mu, p)$ (alpha-stable or mixed alpha-stable distributed)); $a$ is an annuity rate, and $\gamma$ is payment to the fund $\gamma \in\{0 ; 2 ; 3.5 ; 3+1 ; 4+2 ; 5.5+$ 2) percent from the gross salary (see Figure 1).

\subsubsection{Payout from pillar I}

$x_{I, S}$ may be pre-calculated by using the calculator given by SODRA (State social insurance fund) [18]. Currently, the payout from pillar I is calculated by formula (2)

$$
x_{I, s}=\left\{\begin{array}{c}
b p+a p\left(w r, M S_{s}\right), \text { if } \quad w r<30 \\
1.1 \cdot b p+a p\left(w r, M S_{s}\right)+s p(w r-30), \quad \text { otherwise. }
\end{array}\right.
$$

Here $b p$ represents the base pension (managed by the Ministry of Social Security), ap the additional pension, $s p$ the supplementary pension and $M S_{s}$ is the average salary considered as stochastic since influenced by economic conditions. The participant's decisions can affect this payout very weakly. In order to obtain this, one should change the job or work many years to get a higher pension.

Theoretically, pillar I is always available. However, if the country goes in default, the payoff from pillar I will be equal to $0, x_{I, S}=0$. The probability of default for most of the countries is given by credit ratings and risk analysis companies (Moody, S\&P Capital IQ, etc.).

\subsubsection{Payout from pillars II and III}

Payouts from pillars II and III are similar; however, pillar II is more complicated, and we will explain this more widely. $x_{I I, S}$ may be pre-calculated using formula 


$$
x_{I I, s}=A_{w r, s} \cdot \frac{a(1+a)^{n}}{(1+a)^{n}-1},
$$

where $a$ is the annuity rate, $n$ is duration of the annuity contract (life $n=\infty$ or fixed $n=10, \ldots, 25$ duration annuities). The accumulated sum in pillar II $A_{w r, s}$ under a specific scenario $s$, is a solution of the following difference equation

$$
A_{t, s}-\left(1+f r_{t-1, s}\right) A_{t-1, s}=\gamma_{t} \cdot S_{t, s}, \quad i=1, \ldots, w r
$$

with initial conditions $s i_{0, s}=0, \quad A_{0, s}=0, \quad f r_{0, s}=0$. Currently, $\gamma_{0}=2 \%$, but depending on the year (see Figure 1), it will change. The salary in the $i$ th year can be calculated by using formula $S_{t, s}=S_{t-1, s}\left(1+s i_{t, s}\right)$.

The case of not availability of pillar II in the future is expected to be very small. However, the cases of other nations like Poland and Hungary show that this probability is not equal to 0 . Moreover, this is mostly related to a political risk. On the other hand, pension funds are reinsured and in case of default, the payout is not lost.

\subsubsection{Simplified case}

In the simplified single stage of the stochastic optimization model, we suppose that under a specific scenario $s: f r_{t, s} \approx f r_{s}$, i.e., the long-term fund return is constant and approximately equal to the weighted average; $s i_{t, s} \approx s i_{s}$, i.e., the long-term salary increment is constant and approximately equal to the weighted average (the salary increases exponentially). The difference equation is reduced to $A_{t, s}-\left(1+f r_{s}\right) A_{t-1, s}=\gamma \cdot S_{0}\left(1+s i_{s}\right)^{t}$, where $A_{0}$ is the initially accumulated sum in the fund (for participants that have not participated in pillar II before $A_{0}=0$ ), and $t=0, \ldots, w r$ is a year. In this case, equation (4) has a unique solution

$$
A_{t, s}=\left\{\begin{array}{l}
\frac{\gamma\left(1+s i_{s}\right) S_{0}\left[\left(f r_{s}+1\right)^{t}-\left(s i_{s}+1\right)^{t}\right]}{f r_{s}-s i_{s}} \text { if } f r_{s} \neq s i_{s}, \\
\gamma \cdot t \cdot S_{0}\left(f r_{s}+1\right)^{t} \text { if } f r_{s}=s i_{s} .
\end{array}\right.
$$

A simplified version of the problem may be used in most of the cases, especially in the case of single-stage stochastic programming. Since we do not care what will happen in the middle of the working life, we need information only about the fund return at the retirement moment. 
In a simplified case, we can consider the fact that the default of Lithuania in the next 5 years is equal to $9.3 \%$. The probability that pillar II will not be available is very small $(0.1 \%)$. However, possible changes in the pension system could be made in 2016, when the parliamentary elections in Lithuania will be held. It is not certain, but this probability may increase, even if only a small part of population prefers to stop payments to pillar II pension funds (mostly current pensioners).

\subsubsection{Scenario generation}

There are several factors which we have to take into account in order to generate scenarios and build the scenario tree:

- Inflation (influences net salary increment and net returns on conservative fund);

- Condition of economy (crisis, normal, upturn) - influences salary increment and returns on funds:

o Unemployment (influences salary increment);

o Salary (below average, average, higher, very high);

- Political risk (decisions made in the parliament can destroy pillar I or II, Polish experience) - usually not included.

Figure 2 presents the scenario tree considered in general, where the participant at the beginning of work selects the pension fund and works until retirement, paying some amount of money to the same pension fund.

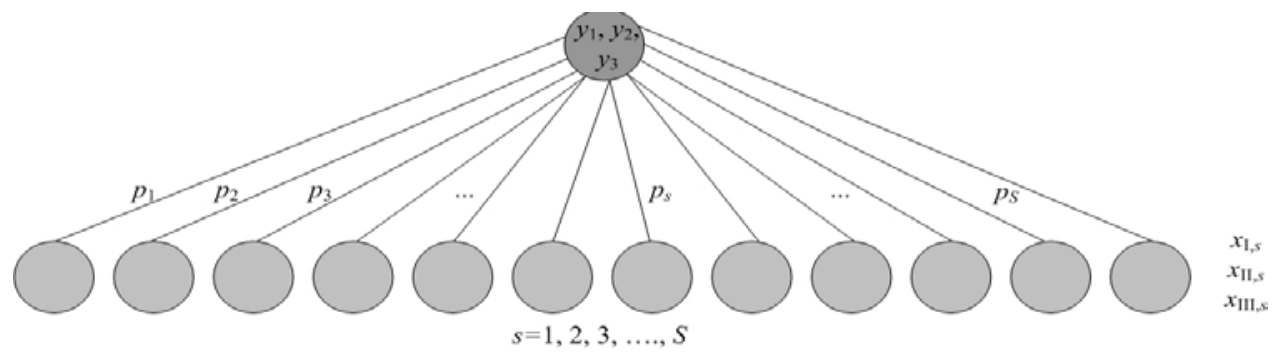

Figure 2: Scenario tree.

The value at the tree nodes (stochastic parameters), with the exception of the root, is a three-dimensional vector $\left(x_{I, S}, X_{I I, s}, x_{I I I, s}\right)$, representing the payout during the period from the moment of decision making to the moment of retirement.

Values $x_{I, s}, X_{I I, s}$ and $x_{I I I, S}$ are calculated using their definitions as described in the previous subsections. 
Probability $p_{s}$ of scenario $s$ is calculated as the probability of the intersection of the following events obtained by considering historical data:

1. type of participant's fund:

$B=\{$ conservative, small stock, medium stock, stock $\}$,

2. economic conditions: $C=$ \{crisis, normal, upturn $\}$,

3. availability of pillar I: $D=\{$ yes, no $\}$,

4. availability of pillar II: $E=\{$ yes, no $\}$,

5. other factors.

Consequently, we can write:

$$
p_{s}=p(B \cap C \cap D \cap E \cap \ldots) .
$$

Events $B \cap C$ and $D \cap E$ are usually independent or (in a simplified case) we can assume them to be independent of each other and of any other event on the list. Then

$$
p_{s}=p(B \cap C) \cdot p(D \cap E) \cdot \ldots
$$

Notice that the scenario with both events $D$ and $E$ with value 'no' has null probability $p(D \mid E)=p($ no $\mid$ no $)=0$ since at least one of the pillars I and II must be available all the time.

Probability $p(B)$ of the type of the participant's fund is obtained from Table 2 as statistical probability. In practice, they are time dependent, because the choices depend on market conditions. However, in a simplified setting we assume them to be constant.

Probabilities $p(C)$ are calculated as a probability of the economic situation during the work life of the participant (or several time periods in a multistage setting).

Probability of upturn $p(C=$ upturn $)$ is calculated as

$$
p(C=\text { upturn })=\frac{t_{u}}{T},
$$

where $T$ is the length of the time period analysed and $t_{u}$ is the length of the time period with economy above a given average level.

The probability of normal situation $p(C=$ normal $)$ is calculated in a similar way $p(C=$ normal $)=\frac{t_{n}}{T}$, where $t_{n}$ is the length of the time period when economy is "historically typical". The probability of crisis is found from $p(C=$ crisis $)=\frac{t_{c}}{T}$, where $t_{c}$ is the length of the time period when economy is in recession or crisis. Time lengths $t_{u}, t_{n}$ and $t_{c}$ are measured in days and calculated from some global or local indices (OMX Baltic Benchmark, S\&P 500, etc.).

For example, the economic situation in Lithuania in the period from 2004 to 2007 ( 1,000 days) was in upturn; from 2007 to 2009 (700 days) it was in crisis; in the period 2009-2011 (700 days) it was in slow upturn, and from 2011 
up to now (2014) the situation is typical (1,000 days). From the historical data in the period considered we get: $T=3400, t_{u}=1700, t_{n}=1000$, and $t_{c}=700$. Finally, probability of upturn $p(C=$ upturn $)=\frac{1700}{3400}=0.5$. However, if more precise results are necessary, one can use some comparative indices to measure time lengths for each fund group separately. In this case, probabilities $p(C)$ would be dependent on probabilities $p(B)$.

\section{Illustrative example}

In this section, we present preliminary numerical results in case of a 19 years old participant, male, who started to work at the end of 2013. The average Lithuanian salary in 2014 was $S_{0}=724.05 €$ and we set the percent from the gross salary payment to the fund at $\gamma=2 \%$ (case B in Figure 1).

For simplicity, as a preliminary case study we consider only 4 different scenarios, according to the type of participant's fund: conservative, small stock, medium stock and stock. We will denote later the considered scenario by $s$ $\in\{$ cons, small, medium, stock $\}$. We consider the case of availability of both pillars I and II. For simplicity, the economic condition is treated as a constant. A better description of the stochasticity by means of a larger number of scenarios will be addressed in future work.

We set the salary increment for all considered scenarios to zero, $s i_{s}=0$, and the annual average return from the pension fund in the four scenarios is $f i_{\text {cons }}=5.06 \%, f i_{\text {small }}=5.34 \%, f i_{\text {medium }}=2.63 \%$ and $f i_{\text {stock }}=-2.74 \%$.

The work record is expected to be 47 years, so $w r=47$, and because of the assumption of zero salary increment, the mean salary $M S=S_{0}=724.05 €$. Consequently, since the economic condition is treated here as deterministic, the payout from pillar I, computed according to [18] and formula (2), is deterministic as well, and is then expected to be $x_{I}=338.28 €$. Furthermore, we assume that the duration of the annuity contract is $n=15$ years with an annuity rate $a=7 \%$. According to formula (3), the payout from pillar II in the 4 scenarios considered is then expected to be: $x_{I I, \text { cons }}=477.94 €$, $x_{I I, \text { small }}=496.01 €, x_{I I, \text { medium }}=370.77 €$ and $x_{I I, \text { stock }}=286.49 €$. Since pillar III is optional, in this preliminary case study we set $y_{3}=0$. Probabilities specified in Section 3.2.4 are as follows: $p_{\text {cons }}=0.0974, p_{\text {small }}=0.2494, p_{\text {medium }}=$ 0.5302 and $p_{\text {stock }}=0.123$. Values of $c_{2}$ and $c_{3}$ are supposed to be zero.

The single-stage stochastic programing problem (1) is then solved under AMPL environment with the Gurobi solver. An optimal solution to problem (1) is as follows: $y_{1}=0, y_{2}=1$, and $y_{3}=0$ with an objective value function of $402.08 €$ which represents the expected total pension for a participant choosing 
pillar II. The payout with respect to the choice of pillar I is approximately $16 \%$ bigger.

\section{Discussion and future work}

In this paper, the pension system of Lithuania is discussed. Special attention is paid to the development of a model which might be used to obtain the payout from pillar II pension funds. The structure of the payout is described and explained in detail. A simplified mathematical model of the pension accrual is given. The described linear single stage stochastic problem allows modelling of the payout from the Lithuanian pension funds. The proposed procedure has not been implemented yet. However, we plan to present and discuss the solution of the approach introduced here in a future paper.

The analysis of the literature and a-priori assumptions suggest that pillar II (case $\mathrm{C}$ from Figure 1) will give a maximal payout. The illustrative example above also shows that the average participant should choose pillar II of the pension system. In that case he/she may expected to have a pension $16 \%$ bigger comparing to the pension from pillar I only. However, Kilianova and Pflug [11] have shown that better performance of pillar II pension funds is achieved by using dynamic management. Such modification of the model described in this paper will be explored in the future. Additional research should be carried out in the generation of the scenario tree with inter-stage dependent coefficients if a multistage stochastic program is designed.

The proposed model can be used in other countries, e.g., Latvia and Estonia. However, the model depends too much on the rules adopted by the governments of each single country and prior to the proposed model application, restrictions of the law must be taken into account.

\section{Acknowledgement}

The authors express their acknowledgments to the Research Council of Lithuania for the support in delivering a cycle of lectures "Stochastic programming and its applications to energy and logistics problems" at Kaunas University of Technology in 2013. 


\section{References}

[1] Bank of Lithuania. (2013). Reviews of Lithuania's 2nd and 3rd pillar pension funds and of the market of collective investment.

https://www.lb.1t/reviews_of_lithuanias_2nd_and_3rd_pillar_pension_funds_a nd_of_the_market_of_cellective_investment_undertakings_1 [Accessed on 23 September 2014].

[2] Birge, J. R., Louveaux, F. (2011). Introduction to Stochastic Programming. Springer-Verlag, New York.

[3] Bitinas, A., Maccioni, A.F. (2014). Lithuanian pension system's reforms transformations and forecasts. Universal Journal of Industrial and Business Management, 2(1), 13-23.

[4] Cai J, Ge C (2012) Multi-objective private wealth allocation without subportfolios. Economic Modelling, 29(3), 900-907.

[5] Consigli, G., Iaquinta, G., Moriggia, V., Tria, M.D., Musitelli, D. (2012). Retirement planning in individual asset-liability management. IMA Journal Management Mathematics, 23(4), 365-396.

[6] Department of Statistics. (2013). Average monthly earnings by sector, year. http://db1. stat.gov.1t/statbank/SelectVarVal/Define. asp?Maintable=M3060801 \&PLanguage $=0$ [Accessed on 23 September 2014].

[7] Department of Statistics. (2013). Minimum monthly and hourly wages by category of employees, month.

http://db1.stat.gov.lt/statbank/SelectVarVal/saveselections.asp [Accessed on 23 September 2014].

[8] Gudaitis, T. (2012). Applying investment management models into fully funded pension system [in Lithuanian]. http://jmk.vvf.vgtu.1t/index.php/conference/2012/paper/viewFile/14/61 [Accessed on 23 September 2014].

[9] Gülpinar, N., Rustem, B., Settergren, R. (2004). Simulation and optimization approaches to scenario tree generation. Journal of Economic Dynamics \& Control, $28,1291-1315$.

[10] Heitsch, H., Romisch W. (2005). Scenario tree modeling for multistage stochastic programs. Mathematics for Key Technologies, DFG Research Center MATHEON, Berlin, Germany, Preprint, 296.

[11] Kilianova, S., Pflug., G.Ch. (2009). Optimal pension fund management under multiperiod risk minimization. Annals of Operational Reserch, 166, 261-270.

[12] Konicz, A.K., Pisinger, D., Rasmussen, K. M., Steffensen, M. (2013). A combined stochastic programming and optimal control approach to personal finance and pensions.

http://www.staff.dtu.dk/agko/Research/ /media/agko/konicz_combined.ashx [Accessed on 23 September 2014].

[13] Lazutka, R. (2007). Development of pension schemes in Lithuania [in Lithuanian]. Filosofija. Sociologija, 18( 2), 64-80.

[14] Maggioni, F., Allevi, E., Bertocchi, M. (2014). Bounds in multistage linear stochastic programming. Journal of Optimization, Theory and Applications, 163(1), 200-229. 
[15] Maggioni, F., Wallace, W. S. (2012). Analyzing the quality of the expected value solution in stochastic programming. Annals of Operations Research, 200(1), 37-54

[16] Mitra, S. (2006). A white paper on scenario generation for stochastic programming. WwW.optirisk-systems.com/papers/SGwhitepaper.pdf [Accessed on 23 September 2014].

[17] Rudyte, D., Beržinskienè, D. (2012). Model of the Lithuanian pension system: Challenges and opportunities. Socialiniai Tyrimai, 2(27), 29-35.

[18] SODRA (State social insurance fund) (2010). Old-Age Pension. http://sena.sodra.1t/index.php?cid=1946 [Accessed on 23 September 2014].

[19] Sutiene, K., Kabasinskas, A., Strebeika, D., Kopa, M., Reichardt, R. (2014). Estimation of $\mathrm{VaR}$ and $\mathrm{CVaR}$ from financial data using simulated alpha-stable random variables. To appear in: Proceedings of the 28th European Simulation and Modelling Conference - ESM'2014, October 22-24, 2014, FEUP - University of Porto, Porto, Portugal. 